\title{
RNA fragment assembly with experimental restraints
}

\author{
Grzegorz Chojnowski ${ }^{1,3, \dagger, *}$, Rafał Zaborowski ${ }^{1,4, \dagger}$, Marcin Magnus ${ }^{5}$, and Janusz M. \\ Bujnicki ${ }^{1,2}$
}

\author{
${ }^{1}$ International Institute of Molecular and Cell Biology, Trojdena 4, 02-109 Warsaw, Poland \\ ${ }^{2}$ Institute of Molecular Biology and Biotechnology, Faculty of Biology, Adam Mickiewicz University, Umultowska 89, \\ 61-614 Poznan, Poland \\ ${ }^{3}$ European Molecular Biology Laboratory, c/o DESY, Notkestrasse 85, 22607 Hamburg, Germany \\ 4Present address: Faculty of Mathematics, Informatics, and Mechanics, University of Warsaw, Banacha 2, 02-097 \\ Warsaw, Poland \\ ${ }^{5}$ ReMedy-International Research Agenda Unit, Centre of New Technologies, University of Warsaw, 02-097 \\ Warsaw, Poland \\ tThese authors contributed equally to this work \\ *To whom correspondence should be addressed.
}

\begin{abstract}
We present RNA Masonry, a computer program and a web service for a fully automated assembly of RNA fragments into geometrically plausible models fulfilling user-provided secondary structure constraints and restraints on tertiary contacts and Small Angle X-ray Scattering (SAXS) data. We illustrate the method description with its recent application to structural studies of viral RNAs with SAXS restraints. The program web server is available at http://iimcb.genesilico.pl/rnamasonry.
\end{abstract}

Contact: gchojnowski@embl-hamburg.de

\section{Introduction}

Non-coding RNAs (ncRNAs) are involved in the regulation of many cellular processes. New families of ncRNAs are being continuously discovered (Kalvari et al. 2018; Boccaletto et al. 2018). In principle, the function of newly characterized ncRNAs could be deciphered from their structure in an approach that proved very successful for proteins (Miao et al. 2015; Doudna 2000). The experimental structure determination of ncRNAs (e.g., by X-ray crystallography, nuclear magnetic resonance, or cryo-electron microscopy) is however difficult, and only a very small fraction of ncRNA families have high-resolution structures available for at least one member (99 out of 3,016 according to Rfam 14.1). Computational structure prediction methods offer an alternative to experimental structure determination, but the purely theoretical models are often of limited accuracy. One promising approach is the computational modelling with the use of restraints derived from low-resolution experimental data (Magnus et al. 2014). To address this issue we developed RNA Masonry, a computer program for fully automated modelling of RNA molecules by assembly of recurrent 3D motifs, with the use of low-resolution restraints. The 3D motifs (further referred to as fragments) are retrieved from the RNA Bricks database (Chojnowski, Walen, and Bujnicki 2014), which catalogues recurrent substructures observed in experimentally-determined, high-resolution RNA structural models available in the Protein Data Bank (Berman et al. 2000). 
RNA Masonry exploits hierarchical organization of RNA molecules, which are composed of 3D motifs defined at a secondary structure level; double-stranded helices, single-stranded fragments, and various types of loops. During the model assembly, the motifs are used as a whole, which strictly preserves the input secondary structure. The program accepts restraints for long-range tertiary interactions. Additionally, the model building can be restrained with a goodness-of-fit to the experimental small angle X-ray scattering (SAXS) data, which is calculated using FoXS (Schneidman-Duhovny et al. 2013) or CRYSOL (Svergun, Barberato, and Koch 1995).

There are other methods available that can assemble RNA 3D structures from fragments of experimentally determined structures (Jossinet, Ludwig, and Westhof 2010; Popenda et al. 2012) one of them designed specifically for the purpose of modelling with SAXS restraints (Gajda et al. 2013). To the best of our knowledge, however, RNA Masonry is the only tool that combines statistical potential with experimental restraints and uses a regularly updated database of fragments (RNA Bricks is updated weekly, http://genesilico.pl/rnabricks2).

\section{Materials and methods}

The program provides two basic functionalities: 1) de novo fragment assembly and 2) RNA model refinement. The input is either RNA sequence with the secondary structure or a preliminary RNA 3D structural model in the PDB or mmCIF format. The program can use SAXS data as an additional restraint in any format recognized by FoXS and CRYSOL.

\subsection{Input processing}

If the input is an RNA atomic model, the program annotates its secondary structure with ClaRNA (Walen et al. 2014) which is further processed analogously to a secondary structure given directly on input for de novo modelling.

Pseudo-knots are removed from the input secondary structure using K2N (Smit et al. 2008) and stored as additional restraints. The pseudo-knot free secondary structure is subsequently decomposed into structural motifs and encoded as an RNA motif-graph introduced in the RNA Bricks database. Briefly, the graph nodes correspond to RNA structural motifs (doublestranded helices, loops, and single-stranded fragments). The graph edges denote nucleotides shared by two neighbouring motifs. Owing to the absence of pseudo-knots in the basic data structure the motif graphs are trees, albeit not necessarily rooted. Pseudoknots are nonetheless enforced at the level of structural restraints.

\subsection{Selecting RNA 3D fragments}

After building the motif-graph, which is a basic data structure used in RNA Masonry, 3D fragments from RNA Bricks database are assigned to each of its nodes following the secondary structure constraints. The fragments are selected based exclusively on the canonical secondary structure. No sequence information is taken into account. If the number of fragments assigned to a node is fewer than a user-defined threshold (10 by default) additional fragments are generated by circular permutations (e.g., by changing ends of symmetrical, internal loops). If needed, new fragments are also automatically built from smaller ones by introducing insertions using ModeRNA (Rother et al. 2011). Finally, each 
fragment is mutated to the target sequence. Any steric conflicts, unsatisfied pseudo-knot restraints, or implausible geometries introduced at this stage are ignored, but penalized later during fragment assembly steps by the SimRNA (Boniecki et al. 2016) scoring function.

\subsection{Starting RNA model assembly}

If RNA 3D structure is given as an input, it is used as a starting model without any further modifications. Otherwise, the program assembles a starting model with a possibly small number of severe clashes, which readily increases the convergence of the subsequent refinement step.

In principle, the aim is to find a configuration of fragments, assigned to each of the motif-graph nodes, which minimizes the number of clashes in a complete model. A clash occurs when two glycosidic nitrogens (N1 for pyrimidines, N9 for purines) are closer than $6 \AA$ apart. To reduce the combinatorial complexity of the task, the assembly process is initiated from the terminal elements of the structure (the motif graph "leaves") and proceeds iteratively by adding a single node at a time. At each step, from all possible partial models only statistically relevant representatives are selected as described by Hajdin et al. (Hajdin et al. 2010).

\subsection{Model optimization}

The starting model is optimized in a replica exchange Monte-Carlo simulation with a geometric distribution of temperature levels. We use a scoring function that combines SimRNA statistical potential (Boniecki et al. 2016) and SAXS curve goodness-of-fit (if given on input). The longrange tertiary contact restraints can be also included (e.g., to indicate pseudoknots). In a single step, a random motif-graph node is selected. Next, from a set of fragments assigned to the node a random one is selected and inserted into corresponding position in the model. For all motifs except terminal loops, this operation changes conformation of a whole domain in the RNA model. The user-provided secondary structure is preserved at each step. The lowestscore model constructed during the entire simulation is returned to the user.

\section{Structural studies of viral RNAs with SAXS restraints}

We used an early version RNA Masonry for modelling adenovirus virus-associated RNA (VAI) structure with SAXS restraints (Dzananovic et al. 2017). Interestingly, a crystal structure of VA-I has been recently determined and turned out to be inconsistent with the results of scattering experiments with goodness-of-fit parameter $\left(\mathrm{X}^{2}\right)$ estimated using CRYSOL of 2.8 (Hood et al. 2019) (Fig. 1A). We used RNA Masonry to optimize the fit of the structure to the SAXS curve, while preserving its secondary structure. The resulting model fits better not only to the experimental data $\left(X^{2}=1.5\right)$, but also to the ab initio reconstruction obtained independently using DAMMIF (Franke and Svergun 2009) (Fig. 1B). This result supports a hypothesis that in solution and in the absence of crystal-lattice constraints VA-I may be flexible and sample additional conformations, possibly at the expense of the pseudoknot disruption (Hood et al. 2019). 

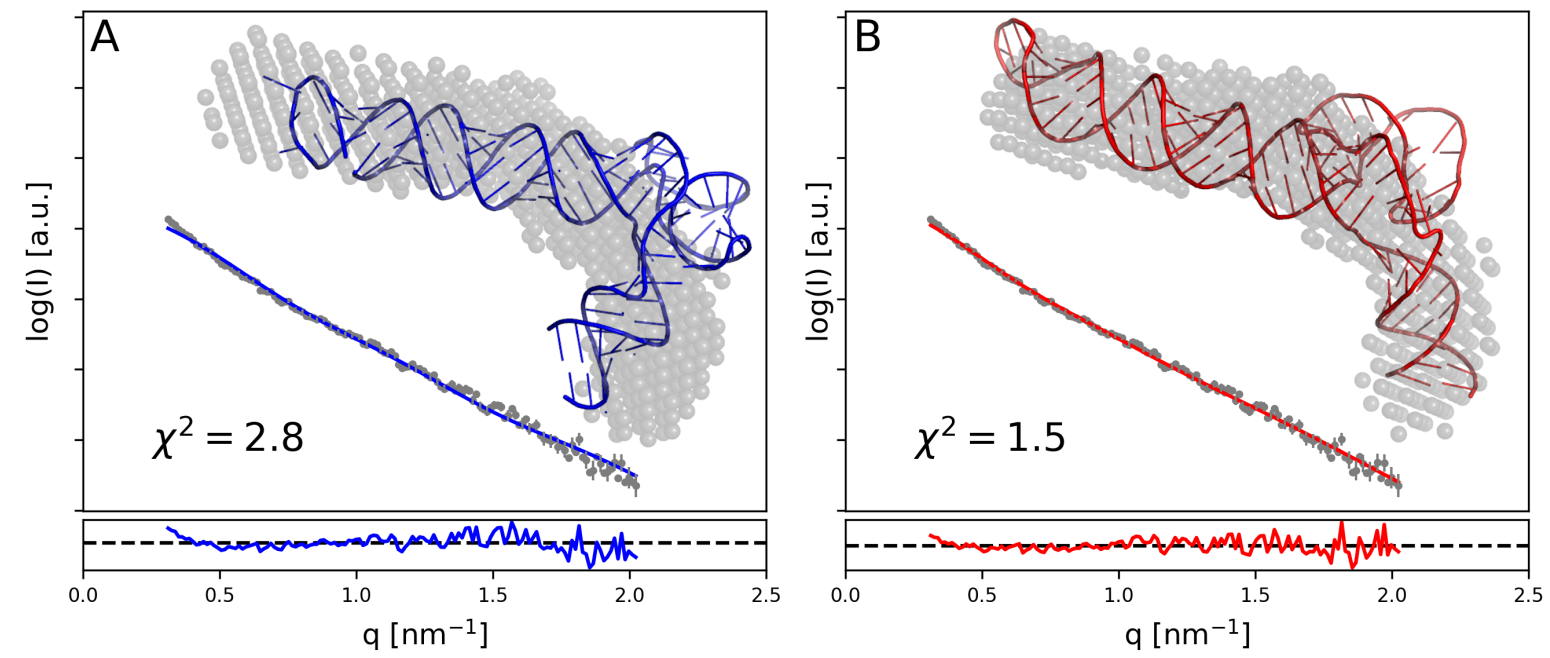

Figure 1 Crystal structure of adenovirus virus-associated RNA (A) was observed to have a conformation inconsistent with results of earlier SAXS experiments. RNA Masonry was used to optimize the fit to experimental SAXS data preserving the crystal structure secondary contacts (B). An ab-initio model obtained with DAMMIF is shown with grey spheres. Residuals of model-calculated and experimental SAXS curve fit are shown in the bottom panels.

\section{Implementation}

RNA Masonry and all utility programs were implemented in Python 2.7 and $\mathrm{C}++$ with an extensive use of routines from the Computational Crystallography Toolbox (cctbx) (GrosseKunstleve et al. 2002), Numpy (Oliphant 2006), Scipy (Virtanen et al. 2020), NetworkX (Hagberg, Swart, and S Chult 2008), Biopython (Cock et al. 2009), ClaRNA, ModeRNA, and K2N libraries. Migration to Python version 3, which requires an update of some of the RNA Masonry dependencies, is planned in due course. The web server engine used in this work is a part of rna-tools toolbox (Magnus et al. 2020) and can be freely reused for new applications.

\section{Conclusions}

RNA Masonry is a computer program for fully automated modelling of RNA 3D structures by fragment assembly. The RNA structure representation used here significantly reduces the number of degrees of freedom, which in principle equals the number of joints (internal loops) in the target structure. The small number of degrees of freedom significantly reduces the modelling time (e.g., compared to SimRNA simulations). In the case of modelling with SAXS data it also reduces the risk of overfitting, given the limited number of unique observations that are generally available from SAXS experiments (Konarev and Svergun 2015). It must be stressed, that at the same time the presented approach is instrinctly unable to model fine details of RNA structures (e.g., non-canonical interactions) that are not encoded within the 3D motifs used for assembly. These, however, can be modeled using complementary highresolution approaches (e.g., using QRNAS (Stasiewicz et al. 2019), SimRNA, or ROSETTA/FARFAR (Yesselman and Das 2016)).

The current RNA Masonry version selects RNA fragments for model-building based exclusively on canonical secondary structure constraints. It was observed, however, that the presence of certain motif types (e.g., kink-turn (Klein et al. 2001)) and coaxial stacking 
between adjacent helices can be reliably predicted from sequence (Cruz and Westhof 2011; Tyagi and Mathews 2007). Both these a priori information could be in principle used to restrain selection of fragments during model building in RNA Masonry to increase reliability of resulting models. We plan such an extension in further releases of the software.

\section{Acknowledgements}

We thank Dr. Michal Boniecki (IIMCB) for sharing the SimRNA scoring function and Dr. Tomasz Waleń (University of Warsaw) for sharing ClaRNA source code.

\section{Funding}

G.C. was supported by the European Research Council (ERC, 261351, grant to J.M.B.). M.M. was supported by the "Regenerative Mechanisms for Health-ReMedy" grant MAB/20172, carried out within the International Research Agendas Program of the Foundation for Polish Science co-financed by the European Union under the European Regional Development Fund. J.M.B. was supported by the Foundation of Polish Science (FNP, TEAM/2016-3/18) and by the Polish National Science Center (NCN, 2017/26/A/NZ1/01083).

\section{Conflict of Interest: none declared}

\section{References}

Berman, H M, J Westbrook, Z Feng, G Gilliland, T N Bhat, H Weissig, I N Shindyalov, and P E Bourne. 2000. "The Protein Data Bank Helen." Nucleic Acids Research 28 (1): 23542. https://doi.org/10.1093/nar/28.1.235.

Boccaletto, Pietro, Marcin Magnus, Catarina Almeida, Adriana Żyła, Astha Astha, Radosław Pluta, Błażej Bagiński, et al. 2018. "RNArchitecture: A Database and a Classification System of RNA Families, with a Focus on Structural Information." Nucleic Acids Research 46 (D1): D202--D205.

Boniecki, Michal J, Grzegorz Lach, Wayne K Dawson, Konrad Tomala, Pawel Lukasz, Tomasz Soltysinski, Kristian M Rother, and Janusz M Bujnicki. 2016. "SimRNA: A Coarse-Grained Method for RNA Folding Simulations and 3D Structure Prediction." Nucleic Acids Research 44 (7): e63--e63.

Chojnowski, G., T. Walen, and J. M. Bujnicki. 2014. "RNA Bricks--a Database of RNA 3D

Motifs and Their Interactions." Nucleic Acids Research 42.D1 (November): D123-31.

https://doi.org/10.1093/nar/gkt1084.

Cock, Peter J A, Tiago Antao, Jeffrey T Chang, Brad A Chapman, Cymon J Cox, Andrew Dalke, Iddo Friedberg, et al. 2009. "Biopython: Freely Available Python Tools for Computational Molecular Biology and Bioinformatics." Bioinformatics 25 (11): 1422-23.

Cruz, José Almeida, and Eric Westhof. 2011. "Sequence-Based Identification of 3D

Structural Modules in RNA with RMDetect." Nature Methods 8 (6): 513-21. https://doi.org/10.1038/nmeth.1603.

Doudna, J. 2000. "Structural Genomics of RNA." Nature Structural Biology 7 (november): 954-56. https://doi.org/10.1038/80729.

Dzananovic, Edis, Grzegorz Chojnowski, Soumya Deo, Evan P Booy, Pauline Padilla-Meier, Kevin McEleney, Janusz M Bujnicki, Trushar R Patel, and Sean A McKenna. 2017. "Impact of the Structural Integrity of the Three-Way Junction of Adenovirus VAI RNA on 
PKR Inhibition." PloS One 12 (10): e0186849.

Franke, Daniel, and Dmitri I Svergun. 2009. "DAMMIF, a Program for Rapid Ab-Initio Shape

Determination in Small-Angle Scattering." Journal of Applied Crystallography 42 (2):

342-46.

Gajda, Michal Jan, Denise Martinez Zapien, Emiko Uchikawa, and Anne-Catherine Dock-

Bregeon. 2013. "Modeling the Structure of RNA Molecules with Small-Angle X-Ray

Scattering Data." PloS One 8 (11): e78007.

https://doi.org/10.1371/journal.pone.0078007.

Grosse-Kunstleve, Ralf W., Nicholas K. Sauter, Nigel W. Moriarty, and Paul D. Adams.

2002. "The Computational Crystallography Toolbox : Crystallographic Algorithms in a

Reusable Software Framework ." Journal of Applied Crystallography 35 (1): 126-36.

https://doi.org/10.1107/s0021889801017824.

Hagberg, Aric, Pieter Swart, and Daniel S Chult. 2008. "Exploring Network Structure,

Dynamics, and Function Using NetworkX."

Hajdin, Christine E, Feng Ding, Nikolay V Dokholyan, and Kevin M Weeks. 2010. "On the

Significance of an RNA Tertiary Structure Prediction." RNA (New York, N.Y.) 16 (7):

1340-49. https://doi.org/10.1261/rna.1837410.

Hood, Iris V, Jackson M Gordon, Charles Bou-Nader, Frances E Henderson, Soheila

Bahmanjah, and Jinwei Zhang. 2019. "Crystal Structure of an Adenovirus Virus-

Associated RNA." Nature Communications 10 (1): 1-12.

Jossinet, Fabrice, Thomas E Ludwig, and Eric Westhof. 2010. "Assemble: An Interactive

Graphical Tool to Analyze and Build RNA Architectures at the 2D and 3D Levels."

Bioinformatics 26 (16): 2057-59.

Kalvari, loanna, Eric P Nawrocki, Joanna Argasinska, Natalia Quinones-Olvera, Robert D

Finn, Alex Bateman, and Anton I Petrov. 2018. "Non-Coding RNA Analysis Using the

Rfam Database." Current Protocols in Bioinformatics 62 (1): e51.

Klein, D J, T M Schmeing, P B Moore, and T A Steitz. 2001. "The Kink-Turn: A New RNA

Secondary Structure Motif." The EMBO Journal 20 (15): 4214-21.

Konarev, Petr V., and Dmitri I. Svergun. 2015. "A Posteriori Determination of the Useful Data

Range for Small-Angle Scattering Experiments on Dilute Monodisperse Systems."

IUCrJ 2: 352-60. https://doi.org/10.1107/S2052252515005163.

Magnus, Marcin, Maciej Antczak, Tomasz Zok, Jakub Wiedemann, Piotr Lukasiak, Yang

Cao, Janusz M Bujnicki, Eric Westhof, Marta Szachniuk, and Zhichao Miao. 2020.

"RNA-Puzzles Toolkit: A Computational Resource of RNA 3D Structure Benchmark

Datasets, Structure Manipulation, and Evaluation Tools." Nucleic Acids Research 48

(2): 576-88.

Magnus, Marcin, Dorota Matelska, Grzegorz Lach, Grzegorz Chojnowski, Michal J Boniecki,

Elzbieta Purta, Wayne Dawson, Stanislaw Dunin-Horkawicz, and Janusz M Bujnicki.

2014. "Computational Modeling of RNA 3D Structures, with the Aid of Experimental

Restraints." RNA Biology 11 (5): 522-36. https://doi.org/10.4161/rna.28826.

Miao, Zhichao, Ryszard W Adamiak, Marc-Frédérick Blanchet, Michal Boniecki, Janusz M

Bujnicki, Shi-Jie Chen, Clarence Cheng, et al. 2015. "RNA-Puzzles Round II:

Assessment of RNA Structure Prediction Programs Applied to Three Large RNA

Structures." RNA (New York, N.Y.) 21 (6): 1066-84.

https://doi.org/10.1261/rna.049502.114.

Oliphant, Travis E. 2006. A Guide to NumPy. Vol. 1. Trelgol Publishing USA.

Popenda, Mariusz, Marta Szachniuk, Maciej Antczak, Katarzyna J Purzycka, Piotr Lukasiak,

Natalia Bartol, Jacek Blazewicz, and Ryszard W Adamiak. 2012. "Automated 3D 
Structure Composition for Large RNAs." Nucleic Acids Research 40 (14): e112--e112.

Rother, Magdalena, Kristian Rother, Tomasz Puton, and Janusz M Bujnicki. 2011.

"ModeRNA: A Tool for Comparative Modeling of RNA 3D Structure." Nucleic Acids

Research 39 (10): 4007-22. https://doi.org/10.1093/nar/gkq1320.

Schneidman-Duhovny, Dina, Michal Hammel, John A. Tainer, and Andrej Sali. 2013.

"Accurate SAXS Profile Computation and Its Assessment by Contrast Variation

Experiments." Biophysical Journal 105 (4): 962-74.

https://doi.org/10.1016/j.bpj.2013.07.020.

Smit, Sandra, Kristian Rother, Jaap Heringa, and Rob Knight. 2008. "From Knotted to

Nested RNA Structures: A Variety of Computational Methods for Pseudoknot Removal."

RNA 14 (3): 410-16. https://doi.org/10.1261/rna.881308.

Stasiewicz, Juliusz, Sunandan Mukherjee, Chandran Nithin, and Janusz M Bujnicki. 2019.

"QRNAS: Software Tool for Refinement of Nucleic Acid Structures." BMC Structural

Biology 19 (1): 5.

Svergun, DIBC, Claudio Barberato, and Michel H J Koch. 1995. "CRYSOL--a Program to

Evaluate X-Ray Solution Scattering of Biological Macromolecules from Atomic

Coordinates." Journal of Applied Crystallography 28 (6): 768-73.

Tyagi, Rahul, and David H Mathews. 2007. "Predicting Helical Coaxial Stacking in RNA Multibranch Loops." Rna 13 (7): 939-51.

Virtanen, Pauli, Ralf Gommers, Travis E Oliphant, Matt Haberland, Tyler Reddy, David Cournapeau, Evgeni Burovski, et al. 2020. "SciPy 1.0: Fundamental Algorithms for

Scientific Computing in Python.” Nature Methods 17 (3): 261-72.

Waleń, Tomasz, Grzegorz Chojnowski, Przemysław Gierski, and Janusz M Bujnicki. 2014.

"ClaRNA: A Classifier of Contacts in RNA 3D Structures Based on a Comparative Analysis of Various Classification Schemes." Nucleic Acids Research 42 (19): e151. https://doi.org/10.1093/nar/gku765.

Yesselman, Joseph D, and Rhiju Das. 2016. "Modeling Small Noncanonical RNA Motifs with the Rosetta FARFAR Server." In RNA Structure Determination, 187-98. Springer. 